\title{
INTERSECTIONS OF HOLOMORPHIC RETRACTS IN BANACH SPACES
}

\author{
MONIKA BUDZYŃSKA and SIMEON REICH ${ }^{\bowtie}$
}

(Received 15 March 2010; accepted 28 October 2010)

Communicated by M. G. Cowling

\begin{abstract}
Using the Kobayashi distance, we provide sufficient conditions for the intersection of a family of holomorphic retracts in a Banach space to also be a holomorphic retract.

2010 Mathematics subject classification: primary 47H10; secondary 32A10, 46G20, 47H09.

Keywords and phrases: complex geodesic, fixed point, holomorphic mapping, holomorphic retract, $k_{D}$-nonexpansive mapping, $k_{D}$-nonexpansive retract, Kobayashi distance.
\end{abstract}

\section{Introduction}

In 1989, Davis and Enflo [7] rediscovered the following fact, which is originally due to Bruck [1]. However, their proof is completely different from that of Bruck.

THEOREM 1.1. Let $C$ be a closed and convex subset of a separable, strictly convex and reflexive Banach space $(X,\|\cdot\|)$, and let $\left\{F_{n}\right\}_{n=1}^{\infty}$ be a sequence of nonexpansive retracts of $C$. If the intersection $\bigcap_{n=1}^{\infty} F_{n}$ is nonempty, then it is a nonexpansive retract of $C$.

In this paper, modifying the Davis-Enflo method, we establish an analogue of this result for the case of an arbitrary family of holomorphic ( $k_{D}$-nonexpansive) retracts (Theorem 5.4 below). We also present an extension of this result to the case of an arbitrary family of nonexpansive retracts (see Theorem 6.3).

\section{The Kobayashi distance and its properties}

Here we recall a few properties of the Kobayashi distance $k_{D}$, which are common to all bounded and convex domains in complex Banach spaces. See [16] for more properties of $k_{D}$. Throughout this section, all Banach spaces $X$ are complex and all domains $D \subset X$ are bounded and convex.

(C) 2010 Australian Mathematical Publishing Association Inc. 1446-7887/2010 \$16.00 
Let $\Delta$ be the open unit disc in the complex plane $\mathbb{C}$. Recall that the Poincaré distance in $\Delta$ is given by

$$
k_{\Delta}(z, w)=\rho_{\Delta}(z, w)=\operatorname{artanh}\left|\frac{z-w}{1-z \bar{w}}\right|=\operatorname{artanh}(1-\tau(z, w))^{1 / 2},
$$

where

$$
\tau(z, w)=\frac{\left(1-|z|^{2}\right)\left(1-|w|^{2}\right)}{|1-z \bar{w}|^{2}} \quad \forall z, w \in \Delta .
$$

Following the usual practice in complex analysis, we call the metric $k_{\Delta}$ a distance, although it is a metric in the topological sense.

Now let $D$ be a bounded and convex domain in a complex Banach space $(X,\|\cdot\|)$. We will use the following definition of the Kobayashi distance $k_{D}$.

Definition 2.1. We define $k_{D}(x, y)$ to be the infimum of all $k_{\Delta}(0, \gamma)$ for which there exists $f \in H(\Delta, D)$ such that $f(0)=x$ and $f(\gamma)=y$.

This is, in fact, the definition of the Lempert function $\delta_{D}$, which was introduced in [18]. The equality $k_{D}=\delta_{D}$ for finite- and infinite-dimensional spaces was proved in [18] and [8], respectively. One can check (see [11]) that the Kobayashi distance $k_{D}$ is locally equivalent to the norm $\|\cdot\|$.

We will also use the following result from [17]. If $x, y, w, z \in D$ and $s \in[0,1]$, then

$$
k_{D}(s x+(1-s) y, s w+(1-s) z) \leq \max \left\{k_{D}(x, w), k_{D}(y, z)\right\} .
$$

Hence each open (closed) $k_{D}$-ball in the metric space $\left(D, k_{D}\right)$ is convex.

Finally, we recall a characterization of $k_{D}$-bounded sets from [11]. Let $D$ be a bounded and convex domain in a complex Banach space $(X,\|\cdot\|)$. A nonempty subset $C$ of $D$ is said to lie strictly inside $D$ if $\inf \{\|x-y\|: x \in C, y \in \partial D\}>0$. In [11], it is shown that a nonempty subset $C$ of $D$ is $k_{D}$-bounded if and only if $C$ lies strictly inside $D$.

\section{The Kobayashi distance and holomorphic mappings}

We begin with the following well-known definition of mappings that are nonexpansive with respect to the Kobayashi distance.

Let $D_{1}$ and $D_{2}$ be bounded and convex domains in the complex Banach spaces $\left(X_{1},\|\cdot\|_{1}\right)$ and $\left(X_{2},\|\cdot\|_{2}\right)$, respectively. A mapping $f: D_{1} \rightarrow D_{2}$ is said to be nonexpansive with respect to the Kobayashi distance if

$$
k_{D_{2}}(f(x), f(y)) \leq k_{D_{1}}(x, y)
$$

for all $x, y \in D$. If $D_{1}=D_{2}=D$, then we say that $f$ is $k_{D}$-nonexpansive.

We observe that each holomorphic mapping $f: D_{1} \rightarrow D_{2}$ is nonexpansive with respect to the Kobayashi distance (see [11]).

We now recall the following basic property of the Kobayashi distance, namely, its lower semicontinuity with respect to the weak topology. 
THEOREM 3.1 (See [15]). Let $X$ be a complex reflexive Banach space and $D \subset X$ be a bounded and convex domain. If $\left\{x_{\alpha}\right\}_{\alpha \in J}$ and $\left\{y_{\alpha}\right\}_{\alpha \in J}$ are nets in D that converge weakly to $x$ and $y$ respectively, and $x, y \in D$, then

$$
k_{D}(x, y) \leq \liminf _{\alpha} k_{D}\left(x_{\alpha}, y_{\alpha}\right) .
$$

As a consequence of the theorem above we obtain the following result.

THEOREM 3.2 (See [3]). Let $D_{1}$ and $D_{2}$ be bounded and convex domains in the complex reflexive Banach spaces $\left(X_{1},\|\cdot\|_{1}\right)$ and $\left(X_{2},\|\cdot\|_{2}\right)$, respectively. If $\left\{f_{\alpha}\right\}_{\alpha \in J}$ is a net of holomorphic mappings $f_{\alpha}: D_{1} \rightarrow D_{2}$ that converges pointwise in the weak topology to a function $f: D_{1} \rightarrow \overline{D_{2}}$ and there exists a point $z_{0} \in D_{1}$ such that $f\left(z_{0}\right) \in D_{2}$, then $f: D_{1} \rightarrow D_{2}$ and $f$ is holomorphic.

We also note an analogous property of $k_{D}$-nonexpansive mappings.

TheOrem 3.3 (See [12]). Let $D_{1}$ and $D_{2}$ be bounded and convex domains in the complex reflexive Banach spaces $\left(X_{1},\|\cdot\|_{1}\right)$ and $\left(X_{2},\|\cdot\|_{2}\right)$, respectively. If $\left\{f_{\alpha}\right\}_{\alpha \in J}$ is a net of nonexpansive (with respect to the Kobayashi distance) mappings $f_{\alpha}: D_{1} \rightarrow$ $D_{2}$ that converges pointwise in the weak topology to a function $f: D_{1} \rightarrow \overline{D_{2}}$ and there exists a point $z_{0} \in D_{1}$ such that $f\left(z_{0}\right) \in D_{2}$, then $f$ also maps $D_{1}$ into $D_{2}$, and is nonexpansive with respect to the Kobayashi distance.

\section{Strict linear convexity in the case of the Kobayashi distance}

If the bounded domain $D$ is strictly convex, that is, $\bar{D}$ is strictly convex in a complex reflexive Banach space $(X,\|\cdot\|)$, then we have more information regarding the linear convexity of balls in $\left(D, k_{D}\right)$.

THEOREM 4.1 (See $[3,4,21,24,25])$. Let $D$ be a bounded and convex domain in a complex reflexive Banach space $(X,\|\cdot\|)$. If $D$ is strictly convex, then each $k_{D}$-ball is also strictly convex in the linear sense.

Hence we have the following definition.

Definition 4.2 (See [5]). Let $D$ be a bounded and convex domain in a complex Banach space $(X,\|\cdot\|)$. We say that the metric space $\left(D, k_{D}\right)$ is strictly linearly convex if each $k_{D}$-ball is strictly convex in the linear sense.

The following example shows that the linear strict convexity of $\left(D, k_{D}\right)$ does not imply the strict convexity of $D$ in a Banach space $(X,\|\cdot\|)$.

EXAMPle 4.3 (See [5]). Consider the domain

$$
D=\Delta \cap\left\{z \in \mathbb{C}: \operatorname{Re} z<\frac{1}{\sqrt{2}}\right\}
$$

in the complex plane $\mathbb{C}$. Then $\left(D, k_{D}\right)$ is strictly convex in the linear sense, but $D$ is not strictly convex in $\mathbb{C}$. 
Using the Bruck method [1, 2], Budzyńska, Kuczumow and Stachura established the following result.

TheOREM 4.4 (See [5]). Let $D$ be a bounded and convex domain in a reflexive Banach space $(X,\|\cdot\|)$. Suppose that the metric space $\left(D, k_{D}\right)$ is strictly linearly convex. Then for every family $\mathcal{F}$ of commuting holomorphic $\left(k_{D}\right.$-nonexpansive $)$ selfmappings of $D$ with a nonempty common fixed point set $\operatorname{Fix}(\mathcal{F})$, the set $\operatorname{Fix}(\mathcal{F})$ is a holomorphic ( $k_{D}$-nonexpansive) retract of $D$.

Under the assumptions on $\left(D, k_{D}\right)$ of this theorem, the fixed point set of each holomorphic ( $k_{D}$-nonexpansive) mapping $f \in \mathcal{F}$ is a holomorphic retract (see [5], and also [19, 20] for a general result in the case of reflexive spaces). Our main result (Theorem 5.4) shows that the theorem above remains valid even without the assumption that the mappings commute.

\section{The family of holomorphic retracts and the family of $k_{D}$-nonexpansive retracts}

Here we use modifications of certain arguments due to Davis and Enflo (see [7], and also Kirk's paper [13]). We begin with the following definition of a strict retraction (see also [22]).

Definition 5.1 (See [7]). Let $D$ be a bounded and convex domain in a complex Banach space $(X,\|\cdot\|)$. If $R: D \rightarrow F$ is a holomorphic $\left(k_{D}\right.$-nonexpansive $)$ retraction of $D$ onto $F$, where $\emptyset \neq F \subset D$, and

$$
k_{D}(R(x), y)<k_{D}(x, y)
$$

whenever $y \in F$ and $x \in D \backslash F$, then $R$ is said to be a strict holomorphic $\left(k_{D^{-}}\right.$ nonexpansive) retraction of $D$ onto $F$.

We need the following technical lemma.

LEMMA 5.2. If $D$ is a bounded and convex domain in a complex Banach space $(X,\|\cdot\|),\left(D, k_{D}\right)$ is strictly convex in the linear sense, $\emptyset \neq F \subset D$ and $R: D \rightarrow F$ is a holomorphic ( $k_{D}$-nonexpansive) retraction of $D$ onto $F$, then the mapping $r: D \rightarrow F$, defined by

$$
r(x)=R\left(\frac{x+R(x)}{2}\right)
$$

for all $x \in D$, is a strict holomorphic ( $k_{D}$-nonexpansive) retraction of $D$ onto $F$.

PROOF. We observe that for all $y \in F$ and $x \in D \backslash F$,

$$
\begin{aligned}
k_{D}(r(x), y) & =k_{D}(R((x+R(x)) / 2), R(y)) \leq k_{D}((x+R(x)) / 2, y) \\
& <\max \left\{k_{D}(x, y), k_{D}(R(x), y)\right\}=k_{D}(x, y),
\end{aligned}
$$

as required. 
Before formulating the second lemma, which is crucial in our subsequent considerations, we introduce the following notions and notation.

Let $D$ be a bounded and convex domain in a complex reflexive Banach space $(X,\|\cdot\|)$. Assume that $\mathcal{F}$ is a family of holomorphic $\left(k_{D}\right.$-nonexpansive $)$ retracts of $D$ indexed by a closed interval of ordinals, that is, $\mathcal{F}=\left\{F_{\alpha}\right\}_{\alpha \leq \gamma}$. Assume that $\aleph_{0} \leq \gamma$. To each limit ordinal $\beta$ such that $\aleph_{0} \leq \beta \leq \gamma$, we associate a fixed ultranet $\left\{\tilde{\alpha}_{\xi, \beta}\right\}_{\xi}$ such that $\tilde{\alpha}_{\xi, \beta}<\beta$ and $\lim _{\xi} \tilde{\alpha}_{\xi, \beta}=\beta$. Let $\emptyset \neq F_{\alpha} \subset D$ and let $r_{\alpha}: D \rightarrow F_{\alpha}$ be a strict holomorphic ( $k_{D}$-nonexpansive) retraction of $D$ onto $F_{\alpha}$ when $1 \leq \alpha \leq \gamma$. Observe that for each $x \in D$ and $y \in F$, all points $r_{\alpha}(x)$ are in the closed $k_{D}$-ball $B\left(y, k_{D}(y, x)\right)$, which is linearly convex and lies strictly inside $D$. This ball is weakly compact because $X$ is reflexive. Hence we can define a net $\left\{p_{\alpha}(x)\right\}_{1 \leq \alpha \leq \gamma}$ of holomorphic ( $k_{D}$-nonexpansive) mappings in the following way. When $\alpha=1$, we set $p_{1}=r_{1}$ and then set

$$
p_{m+1}(x):=\left(r_{m+1} \circ p_{m}\right)(x)
$$

when $1 \leq m \in \mathbb{N}$. If $\alpha \leq \gamma$ and $p_{\tilde{\alpha}}(x)$ is defined when $\tilde{\alpha}<\alpha$, then we set

$$
p_{\alpha}(x):=p_{\beta}(x)=\left(r_{\beta} \circ\left(\underset{\xi}{\operatorname{weak}} \lim p_{\tilde{\alpha} \xi, \beta}\right)\right)(x)
$$

in the case where $\alpha=\beta$ is a limit ordinal, and

$$
p_{\alpha}(x):=\left(r_{\alpha} \circ p_{\alpha-1}\right)(x)=\left(r_{\beta+m} \circ p_{\beta+m-1}\right)(x)
$$

when $\alpha=\beta+m$, where $\beta$ is a limit ordinal and $1 \leq m \in \mathbb{N}$.

LEMMA 5.3. If $F=\bigcap\left\{F_{\alpha}: 1 \leq \alpha \leq \gamma\right\} \neq \emptyset$, then for each $y \in F$ and $x \in D \backslash F$,

$$
k_{D}\left(p_{\gamma}(x), y\right)<k_{D}(x, y) .
$$

PROOF. Each $p_{\alpha}$ is a holomorphic $\left(k_{D}\right.$-nonexpansive) mapping,

$$
k_{D}\left(p_{\alpha}(x), y\right) \leq k_{D}(x, y),
$$

and

$$
k_{D}\left(p_{\alpha^{\prime}}(x), y\right) \leq k_{D}\left(p_{\alpha}(x), y\right)
$$

whenever $1 \leq \alpha<\alpha^{\prime} \leq \gamma, x \in D$ and $y \in F$. Let $\alpha_{0}$ be the first ordinal such that $x \notin \bigcap_{\alpha=1}^{\alpha_{0}} F_{\alpha}$. If $\alpha_{0}=1$, then $x \notin F_{1}$ and

$$
k_{D}\left(p_{\gamma}(x), y\right) \leq k_{D}\left(p_{1}(x), y\right)=k_{D}\left(r_{1}(x), y\right)<k_{D}(x, y) .
$$

If $\alpha_{0}>1$, then $x \in F_{\alpha}$ when $1 \leq \alpha<\alpha_{0}$ and $x \notin F_{\alpha_{0}}$, and therefore $p_{\alpha_{0}}(x)=r_{\alpha_{0}}(x)$, $k_{D}\left(r_{\alpha_{0}}(x), y\right)<k_{D}(x, y)$ and, finally,

$$
k_{D}\left(p_{\gamma}(x), y\right) \leq k_{D}\left(p_{\alpha_{0}}(x), y\right)=k_{D}\left(r_{\alpha_{0}}(x), y\right)<k_{D}(x, y) .
$$

We are now able to formulate and prove the main result of our paper. 
THEOREM 5.4. Let $D$ be a bounded and convex domain in a complex reflexive Banach space $(X,\|\cdot\|)$. If $\left(D, k_{D}\right)$ is strictly convex in the linear sense, $\mathcal{F}$ is a family of holomorphic ( $k_{D}$-nonexpansive) retracts of $D$ and $F=\bigcap_{\tilde{F} \in \mathcal{F}} \tilde{F} \neq \emptyset$, then $F$ is a holomorphic ( $k_{D}$-nonexpansive) retract of $D$.

PROOF. We can assume that $\mathcal{F}$ is a family of holomorphic ( $k_{D}$-nonexpansive) retracts of $D$ indexed by a closed interval of ordinals, that is, $\mathcal{F}=\left\{F_{\alpha}\right\}_{\alpha \leq \gamma}$, where $\gamma$ is a limit ordinal. This is possible after eventually repeating $F_{1}$ at the end a suitable number of times. Let $\Omega$ denote the first uncountable ordinal. To each limit ordinal $\beta$ such that $\aleph_{0} \leq \beta \leq \max \{\gamma, \Omega\}$, we associate a fixed ultranet $\left\{\tilde{\alpha}_{\xi, \beta}\right\}_{\xi}$ such that $\tilde{\alpha}_{\xi, \beta}<\beta$ and $\lim _{\xi} \tilde{\alpha}_{\xi, \beta}=\beta$.

Let $r_{\alpha}$ be a holomorphic ( $k_{D}$-nonexpansive) retraction of $D$ onto $F_{\alpha}$ whenever $1 \leq \alpha \leq \gamma$. By Lemma 5.2, we may assume that all $r_{\alpha}$ are strict retractions. Define a net $\left\{p_{\alpha}(x)\right\}_{\alpha \leq \gamma}$ of holomorphic ( $k_{D}$-nonexpansive) mappings as in Lemma 5.3.

For simplicity of notation, we denote the last mapping $p_{\gamma}$ by $\Phi$ and then introduce the net $\left\{\Phi_{\alpha}(x)\right\}_{\alpha \leq \Omega}$ of iterations of the mapping $\Phi$ by setting, for each $x \in D$,

$$
\Phi_{1}(x):=\Phi(x) \text { and } \Phi_{m+1}(x):=\Phi \circ \Phi_{m}(x)
$$

when $1 \leq m \in \mathbb{N}$,

$$
\Phi_{\alpha}(x):=\Phi_{\beta}(x)=\underset{\xi}{\operatorname{weak}} \lim \Phi_{\tilde{\alpha}_{\xi, \beta}}(x),
$$

provided that $\alpha=\beta$ is a limit ordinal and $\Phi_{\tilde{\alpha}}(x)$ has been defined whenever $\tilde{\alpha}<\beta$, and finally,

$$
\Phi_{\alpha}(x):=\left(\Phi \circ \Phi_{\alpha-1}\right)(x)=\left(\Phi \circ \Phi_{\beta+m-1}\right)(x)
$$

when $\alpha=\beta+m$, where $\beta$ is a limit ordinal and $1 \leq m \in \mathbb{N}$.

Each $\Phi_{\alpha}$ is a holomorphic ( $k_{D}$-nonexpansive) mapping,

$$
k_{D}\left(\Phi_{\alpha}(x), y\right) \leq k_{D}(x, y),
$$

and

$$
k_{D}\left(\Phi_{\alpha^{\prime}}(x), y\right) \leq k_{D}\left(\Phi_{\alpha}(x), y\right)
$$

whenever $1 \leq \alpha<\alpha^{\prime} \leq \gamma, x \in D$ and $y \in F=\bigcap_{\tilde{F} \in \mathcal{F}} \tilde{F}$. Taking into account the fact that the set of all countable limit ordinals is uncountable, for a fixed $y \in F$ we obtain a limit ordinal $\beta$ such that $1<\beta<\Omega$ and

$$
k_{D}\left(\Phi_{\beta+m}(x), y\right)=k_{D}\left(\Phi_{\beta}(x), y\right)
$$

whenever $1 \leq m \in \mathbb{N}$. But this implies that $\Phi_{\beta}(x) \in F$. This means, in turn, that the net $\left\{\Phi_{\alpha}(x)\right\}_{1 \leq \alpha<\Omega}$ is eventually constant. Setting

$$
R(x):=\Phi_{\Omega}(x)=\lim _{\alpha} \Phi_{\alpha}(x)=\Phi_{\beta}(x),
$$

we obtain a holomorphic ( $k_{D}$-nonexpansive) retraction $R: D \rightarrow F$ and this completes the proof.

In order to formulate a more general result, we need the concept of a norming set. 
Definition 5.5 (See [9]). Let $(X,\|\cdot\|)$ be a complex Banach space and $\mathcal{N}$ be a nonempty subset of its dual $X^{*}$. If there exist positive constants $c$ and $C$ such that

$$
\sup \{|l(x)|: l \in \mathcal{N},\|l\| \leq C\} \geq c\|x\|
$$

for each $x \in X$, then we say that $\mathcal{N}$ is a norming set for $X$.

It is obvious that a norming set generates a Hausdorff linear topology $\sigma(X, \mathcal{N})$ on $X$, which is weaker than the weak topology $\sigma\left(X, X^{*}\right)$.

THEOREM 5.6 (See [12]). Let $(X,\|\cdot\|)$ be a complex Banach space, $\mathcal{N}$ be a norming set for $X$, and $D \subset X$ be a bounded and convex domain whose norm closure $\bar{D}$ is compact in $\sigma(X, \mathcal{N})$. If $\left\{x_{\alpha}\right\}_{\alpha \in J}$ and $\left\{y_{\alpha}\right\}_{\alpha \in J}$ are nets in $D$ that converge in $\sigma(X, \mathcal{N})$ to $x$ and $y$ respectively, and $x, y \in D$, then

$$
k_{D}(x, y) \leq \liminf _{\alpha} k_{D}\left(x_{\alpha}, y_{\alpha}\right) .
$$

As a simple consequence of this property of the Kobayashi distance and the characterization of $k_{D}$-boundedness we get the following compactness result.

THEOREM 5.7 (See [12]). Let $(X,\|\cdot\|)$ be a complex Banach space, $\mathcal{N}$ be a norming set for $X$, and $D \subset X$ be a bounded and convex domain whose norm closure $\bar{D}$ is compact in $\sigma(X, \mathcal{N})$. Then each closed $k_{D}$-ball $\bar{B}_{k_{D}}$ is compact in $\sigma(X, \mathcal{N})$.

Next we consider nets of mappings.

THEOREM 5.8 (See [12]). Let $D_{1}$ and $D_{2}$ be bounded and convex domains in the complex Banach spaces $\left(X_{1},\|\cdot\|_{1}\right)$ and $\left(X_{2},\|\cdot\|_{2}\right)$ respectively, and let $\mathcal{N}$ be a norming set for $\left(X_{2},\|\cdot\|_{2}\right)$. If $\overline{D_{2}}$ is compact in $\sigma\left(X_{2}, \mathcal{N}\right)$, and $\left\{f_{\alpha}\right\}_{\alpha \in J}$ is a net of holomorphic mappings $f_{\alpha}: D_{1} \rightarrow D_{2}$ that converges pointwise in the topology $\sigma\left(X_{2}, \mathcal{N}\right)$ to a function $f: D_{1} \rightarrow \overline{D_{2}}$ such that $f\left(z_{0}\right) \in D_{2}$ for some $z_{0} \in D_{1}$, then $f: D_{1} \rightarrow D_{2}$ and $f$ is holomorphic.

We also mention a similar property of $k_{D}$-nonexpansive mappings.

TheOREM 5.9 (See [12]). Let $D_{1}$ and $D_{2}$ be bounded and convex domains in the complex Banach spaces $\left(X_{1},\|\cdot\|_{1}\right)$ and $\left(X_{2},\|\cdot\|_{2}\right)$ respectively, and let $\mathcal{N}$ be a norming set for $\left(X_{2},\|\cdot\|_{2}\right)$. If $\overline{D_{2}}$ is compact in $\sigma\left(X_{2}, \mathcal{N}\right)$, and $\left\{f_{\alpha}\right\}_{\alpha \in J}$ is a net of nonexpansive (with respect to the Kobayashi distance) mappings $f_{\alpha}: D_{1} \rightarrow D_{2}$ that converges pointwise in the topology $\sigma\left(X_{2}, \mathcal{N}\right)$ to a function $f: D_{1} \rightarrow \overline{D_{2}}$ and there exists a point $z_{0} \in D_{1}$ such that $f\left(z_{0}\right) \in D_{2}$, then $f$ also maps $D_{1}$ into $D_{2}$ and is nonexpansive with respect to the Kobayashi distance.

We now formulate an extension of Theorem 5.4.

THEOREM 5.10. Let $(X,\|\cdot\|)$ be a complex Banach space, $\mathcal{N}$ be a norming set for $X$, and $D \subset X$ be a bounded and convex domain whose norm closure $\bar{D}$ is compact in $\sigma(X, \mathcal{N})$. If $\left(D, k_{D}\right)$ is strictly convex in the linear sense, $\mathcal{F}$ is a family of holomorphic 
( $k_{D}$-nonexpansive) retracts of $D$ and $F=\bigcap_{\tilde{F} \in \mathcal{F}} \tilde{F} \neq \emptyset$, then $F$ is a holomorphic ( $k_{D}$-nonexpansive) retract of $D$.

Before presenting a simple example of a nonreflexive Banach space for which the theorem above is valid, we recall the notion of the analytic Radon-Nikodym property (ARNP).

We denote by $H^{\infty}(\Delta, X)$ the space of all holomorphic mappings with bounded range from the open unit disc $\Delta$ into a complex Banach space $(X,\|\cdot\|)$, and furnish it with the supremum norm.

Definition 5.11 (See [6]). Let $(X,\|\cdot\|)$ be a complex Banach space. If every $f$ in $H^{\infty}(\Delta, X)$ has radial limits almost everywhere, that is, $\lim _{r \rightarrow 1^{-}} f\left(r e^{i \theta}\right)$ exists for almost all $\theta \in\left([0,2 \pi], \mu_{1}\right)$, where $\mu_{1}$ denotes the Lebesgue measure, then we say that $X$ has the ARNP.

Next we recall the following two theorems.

TheOREM 5.12 (See $[6,10,23])$. All complex reflexive Banach spaces $(X,\|\cdot\|)$ and all separable dual complex Banach spaces $(X,\|\cdot\|)$ have the ARNP.

THEOREM 5.13 (See [4]). Let $(X,\|\cdot\|)$ be a complex Banach space, $\mathcal{N}$ be a norming set for $X$, and $D \subset X$ be a bounded and strictly convex domain whose norm closure $\bar{D}$ is compact in $\sigma(X, \mathcal{N})$. If $X$ has the ARNP, then any $k_{D}$-ball is strictly convex in the linear sense.

We are now ready to present the simple example of a nonreflexive Banach space for which Theorem 5.10 is valid.

EXAMPlE 5.14 (See [4]). Let $\left(\ell_{1},\|\cdot\|_{1}\right)$ be the complex Banach space dual to the complex Banach space $\left(c_{0},\|\cdot\|_{\infty}\right)$, where $\|\cdot\|_{1}$ and $\|\cdot\|_{\infty}$ are the standard norms in $\ell_{1}$ and $c_{0}$, respectively. We introduce in $\ell_{1}$ a new norm,

$$
\|x\|:=\left(\|x\|_{1}^{2}+\sum_{i=1}^{\infty} \frac{\left|x_{i}\right|^{2}}{2^{i}}\right)^{1 / 2}
$$

where $x=\left\{x_{i}\right\} \in \ell_{1}$. Then the complex Banach space $\left(\ell_{1},\|\cdot\|\right)$ is separable and dual to some complex Banach space $\left(Y,\|\cdot\|_{Y}\right)$. Therefore $\left(\ell_{1},\|\cdot\|\right)$ has the ARNP. In addition, it is strictly convex. This implies that, when we take $D$ to be the unit ball of $\left(\ell_{1},\|\cdot\|\right)$ and $\mathcal{N}$ to be $Y$, the assumptions of Theorem 5.10 are satisfied.

The following example shows that the assumption of strict convexity in the linear sense of $\left(D, k_{D}\right)$ in Theorems 5.4 and 5.10 is essential.

EXAmple 5.15. Let $D=\Delta \times \Delta$, where $\Delta$ is the unit open disc. Taking

$$
\begin{gathered}
F_{1}=\left\{\left(z_{1}, z_{2}\right) \in \Delta \times \Delta: z_{2}=\frac{1}{2} z_{1}\right\} \quad \text { and } \quad r_{1}\left(z_{1}, z_{2}\right)=\left(z_{1}, \frac{1}{2} z_{1}\right), \\
F_{2}=\left\{\left(z_{1}, z_{2}\right) \in \Delta \times \Delta: z_{2}=z_{1}^{2}\right\} \quad \text { and } \quad r_{2}\left(z_{1}, z_{2}\right)=\left(z_{1}, z_{1}^{2}\right),
\end{gathered}
$$


for all $\left(z_{1}, z_{2}\right) \in \Delta \times \Delta$, we see that

$$
F_{1} \cap F_{2}=\left\{(0,0),\left(\frac{1}{2}, \frac{1}{4}\right)\right\} .
$$

This means that $F_{1} \cap F_{2}$ is not a holomorphic retract of $\Delta \times \Delta$.

To complete our considerations in this section we recall two basic facts regarding holomorphic ( $k_{D}$-nonexpansive) self-mappings of a bounded and convex domain $D$ for which $\left(D, k_{D}\right)$ is strictly convex in the linear sense.

Lemma 5.16 (See [4]). Let $(X,\|\cdot\|)$ be a complex Banach space, $\mathcal{N}$ be a norming set for $X$, and $D \subset X$ be a bounded and convex domain whose norm closure $\bar{D}$ is compact in $\sigma(X, \mathcal{N})$. Let $\left(D, k_{D}\right)$ be strictly convex in the linear sense. If $f: D \rightarrow D$ is $k_{D}$-nonexpansive and has a fixed point, then $f$ has a fixed point in each nonempty, $f$-invariant, $k_{D}$-closed and convex subset $C$ of $D$.

Using the Bruck method [1], we get the following retraction result.

THEOREM 5.17 (See [4]). Let $(X,\|\cdot\|)$ be a complex Banach space, $\mathcal{N}$ be a norming set for $X$, and $D \subset X$ be a bounded and convex domain whose norm closure $\bar{D}$ is compact in $\sigma(X, \mathcal{N})$. Let $\left(D, k_{D}\right)$ be strictly convex in the linear sense. If $f: D \rightarrow D$ is $k_{D}$-nonexpansive (holomorphic), then $\operatorname{Fix}(f)$ is either empty or a holomorphic $\left(k_{D^{-}}\right.$ nonexpansive) retract of $D$.

\section{The family of nonexpansive retracts in Banach spaces}

In 1970, Bruck [1] announced the following general theorem.

THEOREM 6.1. If $C$ is a closed and convex subset of a strictly convex and reflexive Banach space $(X,\|\cdot\|)$, then a nonempty intersection of any family of nonexpansive retracts of $C$ is also a nonexpansive retract of $C$.

In his proof, Bruck used the strict convexity of the reflexive Banach space $(X,\|\cdot\|)$ in a way which cannot be directly applied to the case of the Kobayashi distance. In 1973, he published another proof of this theorem [2]. In his second proof, he used the fact that each nonempty, bounded and nonexpansive retract in a strictly convex reflexive Banach space is convex, and then applied another of his results, which we now recall [2, Lemma 4(a)].

Lemma 6.2. Let $(X,\|\cdot\|)$ be a Banach space. Suppose that $C$ is a locally weakly compact subset of $X$ and $\left\{F_{\alpha}\right\}$ is a family of weakly closed nonexpansive retracts of $C$, which is directed by set inclusion. If $F=\bigcap_{\alpha} F_{\alpha} \neq \emptyset$, then $F$ is a nonexpansive retract of $C$.

In 2005, Kirk [13] presented a slight modification of the proof of Lemma 6.2. Unfortunately, we are not able to apply either the Bruck method or the Kirk method to the Kobayashi distance case. The reason for this is topological in nature: $k_{D}$-nonexpansive retracts of the strictly linearly convex metric space $\left(D, k_{D}\right)$, where $D$ 
is contained in a reflexive Banach space, need not be convex and therefore we do not know if they are locally weakly closed. But a simple analysis of our proofs leads immediately to the following result. It means, for example, that the concept of a nonexpansive hull of a nonempty subset of a Banach space [14] may be extended beyond reflexive spaces.

THEOREM 6.3. Let $(X,\|\cdot\|)$ be a strictly convex Banach space and $\mathcal{T}$ be a linear topology in $X$. Let the norm $\|\cdot\|$ be lower semicontinuous with respect to $\mathcal{T}$. If $C$ is a convex subset of $X$ that is locally compact in the topology $\mathcal{T}$ (that is, each nonempty intersection of $C$ with a closed ball is compact), then a nonempty intersection of any family of nonexpansive retracts of $C$ is a nonexpansive retract of $C$.

\section{Acknowledgements}

The first author was partially supported by Polish MNiSW grant N N201 393737. The second author was partially supported by the Fund for the Promotion of Research at the Technion and by the Technion President's Research Fund. Part of this research was carried out when the authors visited each other's institutions. They thank the respective departments for their kind hospitality. Both authors are grateful to Tadeusz Kuczumow for many helpful discussions and suggestions.

\section{References}

[1] R. E. Bruck, 'Nonexpansive retracts of Banach spaces', Bull. Amer. Math. Soc. 76 (1970), 384-386.

[2] R. E. Bruck, 'Properties of fixed point sets of nonexpansive mappings in Banach spaces', Trans. Amer. Math. Soc. 179 (1973), 251-262.

[3] M. Budzyńska and T. Kuczumow, 'A strict convexity of the Kobayashi distance', in: Fixed Point Theory and Applications, Vol. 4 (ed. Y. J. Cho) (Nova Science Publishers, Hauppauge, NY, 2003), pp. 27-33.

[4] M. Budzyńska and T. Kuczumow, 'Linear strict convexity of the Kobayashi distance in nonreflexive Banach spaces', in: Fixed Point Theory and its Applications (eds. H. Fetter Nathansky, B. Gamboa de Buen, K. Goebel, W. A. Kirk and B. Sims) (Yokohama Publishers, Yokohama, 2006), pp. 1-9.

[5] M. Budzyńska, T. Kuczumow and A. Stachura, 'Properties of the Kobayashi distance', in: Proceedings of the Second Conference on Nonlinear Analysis and Convex Analysis (eds. W. Takahashi and T. Tanaka) (Yokohama Publishers, Yokohama, 2003), pp. 25-36.

[6] A. V. Bukhvalov and A. A. Danilevich, 'Boundary properties of analytic and harmonic functions with values in a Banach space', Mat. Zametki 31 (1982), 203-214, English translation: Math. Notes 31 (1982), 104-110.

[7] W. J. Davis and P. Enflo, 'Contractive projections on $\ell_{p}$ spaces', in: Analysis at Urbana (Urbana, IL, 1986-1987), Vol. I, London Mathematical Society Lecture Note Series, 137 (Cambridge University Press, Cambridge, 1989), pp. 151-161.

[8] S. Dineen, R. M. Timoney and J.-P. Vigué, 'Pseudodistances invariantes sur les domaines d'un espace localement convexe', Ann. Sc. Norm. Super. Pisa 12 (1985), 515-529.

[9] N. Dunford, 'Uniformity in linear spaces', Trans. Amer. Math. Soc. 44 (1938), 305-356.

[10] C. Grossetête, 'Classes de Hardy et de Nevanlinna pour les fonctions holomorphes à valeurs vectorielles', C. R. Acad. Sci. Paris A-B 274 (1972), A251-A253. 
[11] L. A. Harris, 'Schwarz-Pick systems of pseudometrics for domains in normed linear spaces', in: Advances in Holomorphy, North-Holland Mathematics Studies, 34 (North-Holland, AmsterdamNew York, 1979), pp. 345-406.

[12] J. Kapeluszny and T. Kuczumow, 'A few properties of the Kobayashi distance and their applications', Topol. Methods Nonlinear Anal. 15 (2000), 169-177.

[13] W. A. Kirk, 'Nonexpansive retracts and minimal invariant sets', in: Fixed Point Theory and its Applications (eds. H. Fetter Nathansky, B. Gamboa de Buen, K. Goebel, W. A. Kirk and B. Sims) (Yokohama Publishers, Yokohama, 2006), pp. 161-169.

[14] E. Kopecká and S. Reich, 'Nonexpansive retracts in Banach spaces', Banach Center Publ. 77 (2007), 161-174.

[15] T. Kuczumow, 'The weak lower semicontinuity of the Kobayashi distance and its application', Math. Z. 236 (2001), 1-9.

[16] T. Kuczumow, S. Reich and D. Shoikhet, 'Fixed points of holomorphic mappings: a metric approach', in: Handbook of Metric Fixed Point Theory (eds. W. A. Kirk and B. Sims) (Kluwer Academic Publishers, Dordrecht, 2001), pp. 437-515.

[17] T. Kuczumow and A. Stachura, 'Iterates of holomorphic and $k_{D}$-nonexpansive mappings in convex domains in $\mathbb{C}^{n}$, , Adv. Math. 81 (1990), 90-98.

[18] L. Lempert, 'Holomorphic retracts and intrinsic metrics in convex domains', Anal. Math. 8 (1982), 257-261.

[19] P. Mazet and J.-P. Vigué, 'Points fixes d'une application holomorphe d'un domaine borné dans lui-même', Acta Math. 166 (1991), 1-26.

[20] P. Mazet and J.-P. Vigué, 'Convexité de la distance de Carathéodory et points fixes d'applications holomorphes', Bull. Sci. Math. 116 (1992), 285-305.

[21] G. Patrizio, 'Parabolic exhaustions for strictly convex domains', Manuscripta Math. 47 (1984), 271-309.

[22] S. Reich and A. J. Zaslavski, 'Attracting mappings in Banach and hyperbolic spaces', J. Math. Anal. Appl. 253 (2001), 250-268.

[23] R. Ryan, 'Boundary values of analytic vector valued functions', Indag. Math. 24 (1962), 558-572.

[24] J.-P. Vigué, 'La métrique infinitésimale de Kobayashi et la caractérisation des domaines convexes bornés', J. Math. Pures Appl. 78 (1999), 867-876.

[25] J.-P. Vigué, 'Stricte convexité des domaines bornés et unicité des géodésiques complexes', Bull. Sci. Math. 125 (2001), 297-310.

\section{MONIKA BUDZYŃSKA, Instytut Matematyki UMCS, 20-031 Lublin, Poland e-mail: monikab1@hektor.umcs.lublin.pl}

SIMEON REICH, Department of Mathematics, The Technion-Israel Institute of Technology, 32000 Haifa, Israel e-mail: sreich@tx.technion.ac.il 